\title{
Outstanding Papers from the 2009 Biomedical Engineering Society (BMES) Annual Meeting
}

It is with great pleasure that we recognize the following Outstanding Cellular and Molecular Bioengineering (CMBE) Papers, specially selected from the 2009 BMES Annual Meeting by the Cellular and Molecular Bioengineering Session Chairs of the 2009 BMES meeting and by the CMBE Co-Editors-inChief. We would like to extend our congratulations to the authors of these excellent papers!

(1) Peng Shi, Keyue Shen, Saba Ghassemi, James Hone, and Lance Kam, "Dynamic Force Generation by Neural Stem Cells"

(2) Dimitrijie Stamenovic, Konstatinos A. Lazopoulos, Athanassios Pirentis, and Bela Suki, "Mechanical Stability Determines Stress Fiber and Focal Adhesion Orientation"

(3) Saheli Sarkar, Thomas Egelhoff, and Harihara Baskaran, "Insights Into the Roles of Non-Muscle Myosin IIA in Human Keratinocyte Migration"
The annual BMES meeting continues to grow, this year setting a new record for attendance, with the area of Cellular and Molecular Bioengineering serving as a major part of the growth. To highlight some of the outstanding research presented at the 2009 BMES Annual Meeting, the 2009 BMES Program Committee nominated the outstanding abstracts and invited the authors to submit their findings to CMBE. We now proudly present these peer-reviewed Outstanding Papers from the 2009 BMES Annual Meeting. We would also like to thank the session chairs of the Cellular and Molecular Bioengineering Track of the 2009 BMES Meeting for their help in nominating these Outstanding Papers.

David J. Odde and X. Edward Guo Co-Editors-in-Chief 